REFERENCES

Dunnigan, M. G., Cockrane, M. A., Kelly, A., and Scott, J. W. (1974). Familial lipoatrophic diabetes with dominant transmission. Quarterly fournal of Medicine, 43, 33.

Seip, M. (1959). Lipodystrophy and gigantism with associated endocrine manifestations: a new diencephalic syndrome? Acta Paediatrica, 48, 555.

Senior, B., and Gellis, S. S. (1964). The syndromes of total lipodystrophy and of partial lipodystrophy. Pediatrics, 33, 593.

D. LILLYSTONE and R. J. WEST*

Department of Child Health, Institute of Child Health, 30 Guilford Street, London WC1N 1EH.

^Correspondence to R. J. West, St. George's Hospital, Blackshaw Rd., Tooting, London S.W.17.

\section{Perinatal infections caused by Haemophilus influenzae}

Haemophilus influenzae is occasionally isolated from the female genital tract (Hurley, 1970), and a few cases of perinatal infections involving either the mother or the baby have been reported. Ellner and Shahidi (1969) described 2 cases of puerperal bacteraemia caused by $H$. influenzae, one due to a capsulated type $f$ strain, the other to a rough untyped strain isolated in mixed culture. Though both patients had evidence of endometritis, their babies appeared to be uninfected. The rarity of neonatal infections has been attributed to the passive transfer of protective antibody to the baby, since most adults possess serum bactericidal activity against $H$. influenzae type $\mathrm{b}$ (Fothergill and Wright, 1933). Graber et al. (1971), however, found a much lower incidence of antibody to a strain of $H$. influenzae type $\mathrm{b}$ in both maternal and cord bloods, and considered that this indicated increasing susceptibility of neonates to this organism. These findings were criticized on experimental grounds by Mpairwe (1972), whose own findings accorded with those of Fothergill and Wright (1933). Neonatal infections were reported by Mathies, Hodgman, and Ivler (1965), Collier, Conner, and Nyhan (1967), and Graber et al. (1971), and were all attributed to absence of protective antibody in the maternal sera at the time of delivery. Ingman (1970) described 2 cases of neonatal septicaemia caused by $H$. influenzae; one strain was capsulated type $b$, the other strain was not typed. Both mothers had clinical evidence of endometritis, though in neither case was the organism isolated from the maternal genital tract. Berczy, Fernlund, and Kamme (1973) isolated a noncapsulated strain of $H$. influenzae from the brain of a 22-week-old fetus and subsequently isolated a similar organism from vaginal discharge present in the mother.
Zinner et al. (1972) reported a case of puerperal bacteraemia and neonatal sepsis due to Haemophilus parainfluenzae, supported by immunological studies which indicated that the baby was infected during labour.

In this paper we report 2 further cases of perinatal infection, in which noncapsulated $H$. influenzae was recovered from both mother and baby.

\section{Case reports}

Case 1. A 31-year-old mother of 2 healthy children was admitted to hospital at term after an uncomplicated third pregnancy. For 2 days before labour she noticed a white odourless vaginal discharge, which was not investigated further. Onset of labour occurred some 18 hours after spontaneous rupture of membranes. During labour, the patient developed a temperature of $39.6^{\circ} \mathrm{C}$, accompanied by rigors, but antibiotics were withheld until after delivery. A female infant weighing $3400 \mathrm{~g}$ was delivered by forceps extraction under epidural anaesthesia. The baby gasped after one minute, but regular respirations were not established until 6 minutes, and intubation and intermittent positive pressure ventilation were required initially. At 10 minutes the baby was pink with good tone, but showed tachypnoea. The heart and lungs were clinically normal, the liver and spleen were not enlarged, but the kidneys were easily palpable. Chest $x$-ray showed patchy consolidation in the right mid- and upper zones. The maternal liquor was noticed to be slightly offensive. A clinical diagnosis of neonatal septicaemia was made, and the baby was immediately treated with intravenous penicillin and intramuscular kanamycin.

A blood sample taken from the baby soon after delivery proved sterile on culture. Gastric aspirate showed numerous leucocytes in the Gram-stained deposit, but no organisms were seen and cultures were sterile. The urine was sterile. $H$. influenzae was isolated in pure culture from swabs of umbilicus, ear, nose, and throat, and from the tip of the endotracheal tube. The organism was noncapsulated. The cord blood IgM level was $40 \mathrm{mg} / \mathrm{dl}$.

Susequent progress was satisfactory. The tachypnoea settled within 24 hours, and chest $x$-ray became normal. Jaundice was noticed within the first 24 hours, serum bilirubin rising to $15 \mathrm{mg} / \mathrm{dl}$ on the fifth day. Penicillin was replaced on the fourth day by a 10-day course of ampicillin in view of the bacteriological reports, and the infant appeared normal when examined at 4 weeks.

$H$. influenzae was isolated from the fetal surface of the placenta and from a high vaginal swab taken from the mother soon after delivery. A maternal blood sample taken during labour was sterile on culture. The mother received penicillin and kanamycin after delivery, but $H$. influenzae was still isolated from the vagina several weeks later before treatment was changed to oral ampicillin combined with povidone-iodine pessaries. There was no personal or family history of infections likely to be associated with $H$. influenzae. 
Case 2. An 18-year-old mother with one healthy child was admitted to hospital at 31 weeks' gestation with a mild antepartum haemorrhage. The cervix showed a cystic change accompanied by slight vaginal discharge. She was readmitted at 39 weeks when the height of the fundus suggested a maturity 2 or 3 weeks less than the dates. Spontaneous rupture of membranes was followed 26 hours later by normal delivery.

The infant was a male, birthweight $2230 \mathrm{~g}$, with a maturity assessed at 35 weeks. He was apnoeic at birth, but responded rapidly to intermittent positive pressure ventilation and was pink with spontaneous respirations by 4 minutes. Respiratory distress from birth had persisted at 2 hours of age, and chest $x$-ray showing hazy lung fields and an air bronchogram supported a provisional diagnosis of hyaline membrane disease. Umbilical arterial and venous catheters were inserted at 2 hours, and the arterial $\mathrm{Po}_{2}$ in $100 \%$ oxygen was $140 \mathrm{~mm}$. There was marked metabolic acidosis with an arterial $p \mathrm{H} 7 \cdot 18$ and $\mathrm{PCO}_{2} 19$, which was corrected with intravenous sodium bicarbonate. He deteriorated rapidly, and by 4 hours was apnoeic and required intermittent positive pressure ventilation. $100 \%$ oxygen and gradually increasing ventilation pressures were required to maintain satisfactory arterial blood gases. By 10 hours of age no form of ventilation could maintain an adequate arterial $\mathrm{Po}_{2}$ and he died one hour later.

In view of the rapid progression of respiratory distress and the early severe acidosis, infection was considered. Blood cultures were taken and treatment with penicillin and kanamycin started at 5 hours of age. Blood culture yielded a growth of a noncapsulated strain of $H$. influenzae. No growth was obtained from nasal, umbilical, and rectal swabs. The cord blood IgM level was $<30 \mathrm{mg} / \mathrm{dl}$.

Necropsy showed evidence of hyaline membrane disease, but no pneumothorax, pulmonary haemorrhage, or pneumonia. $H$. influenzae was cultured from the lungs. No other contributory cause of death was found. The placenta showed a small area of infarction, but was otherwise unremarkable. The puerperium was uneventful and the mother was discharged home at 3 days and remained well. A vaginal swab taken from the mother 2 weeks after delivery yielded a heavy, almost pure growth of noncapsulated $H$. influenzae.

\section{Discussion}

It is likely that both mothers were carrying $H$. influenzae in the lower genital tract during pregnancy, and that intrauterine infection occurred by the ascending route after rupture of the membranes. If so, it appears that vaginal colonization or infection does not produce an immune response which is adequate to protect the infant from infection at birth. Similar considerations also apply to neonatal sepsis of acute-onset type caused by group B streptococci (Klesius et al., 1973). An alternative hypothesis is that intrauterine infection occurs by haematogenous spread and that vaginal carriage of $H$. influenzae in the puerperium occurs subsequently. Unfortunately, prenatal vaginal swabs were not available to verify this, but it must be considered uncommon for noncapsulated strains of $H$. influenzae to disseminate in the blood stream.

In Case 1 there was clinical evidence of amnionitis, which probably accounted for the maternal fever and rigors and for the heavy colonization of the baby with $H$. influenzae. Though the baby's blood culture was sterile, the tachypnoea, abnormal chest $x$-ray, early jaundice, and raised cord IgM all pointed to significant neonatal infection. Survival probably depended on maturity and prompt administration of chemotherapy. A similar case report of perinatal infection was described by Ingman (1970), in which an untyped strain of $H$. influenzae was isolated from the baby but not from the mother.

In Case 2 there was no clinical evidence of maternal infection. The baby was premature with severe hyaline membrane disease, and while the bacteraemia could have been a terminal event, the rapid deterioration in the blood gases and the early severe metabolic acidosis suggested that infection may have contributed to death. Delivery was delayed after membrane rupture, and chemotherapy was not initiated until 5 hours after birth.

All available sera were examined for precipitins to organism extracts of $H$. influenzae by the method of Davies, Laughton, and May (1974). Antibodies were not detected in the antenatal specimens of the mothers nor in the cord bloods, and the postnatal serum obtained from the mother in Case 1 at 2 weeks after delivery was also negative. No postnatal specimen was available from the mother in Case 2.

Both infants were treated initially with penicillin and kanamycin. Currently kanamycin or gentamicin has replaced ampicillin as the broad spectrum antibiotic in neonatal infections, but it must be emphasized that they are less effective than ampicillin against $H$. influenzae.

\section{Summary}

Two cases of perinatal infection due to noncapsulated $H$. influenzae are described, in which the organisms were recovered from both mothers and babies. In one case the mother showed clinical signs of infection during labour and the baby survived. In the other, the mother was unaffected but the baby died of severe hyaline membrane disease associated with bacteraemia. The value of ampicillin is stressed. 
We thank Professor J. P. M. Tizard, Dr. H. L. Ellis, Mr. J. D. Ellis, and Mr. John Bonnar for permission to describe patients admitted under their care; also Dr. D. C. Turk, who kindly examined the bacterial isolates, and Dr. J. Faux, who carried out the serological tests.

\section{REFERENCES}

Berczy, J., Fernlund, K., and Kamme, C. (1973). Haemophilus influenzae in septic abortion. Lancet, $1,1197$.

Collier, A. M., Conner, J. D., and Nyhan, W. L. (1967). Systemic infection with Haemophilus influenzae in very young infants. Fournal of Pediatrics, 70, 539.

Davies, J. L., Laughton, C. R., and May, J. R. (1974). An improved test for Haemophilus influenzae precipitins in the serum of patients with chronic respiratory disease. fournal of Clinical Pathology, 27, 265.

Ellner, P. D., and Shahidi, A. (1969). Postpartum bacteremia due to Haemophilus influenzae . Obstetrics and Gynecology, 34, 403.

Fothergill, L. D., and Wright, J. (1933). Influenzal meningitis. fournal of Immunology, 24, 273.

Graber, C. D., Gershanik, J. J., Levkoff, A. H., and Westphal, M. (1971). Changing pattern of neonatal susceptibility to Haemophilus influenzae. Fournal of Pediatrics, 78, 948.

Hurley, R. (1970). Haemophilus endometritis in woman fitted with Lippes Loop. British Medical fournal, 1, 566.

Ingman, M. J. (1970). Neonatal Haemophilus influenzae septicemia originating from material amnionitis. American fournal of Diseases of Children, 119, 66.

Klesius, P. H., Zimmerman, R. A., Mathews, J. H., and Krushak, D. H. (1973). Cellular and humoral immune response to group B streptococci. Fournal of Pediatrics, 83, 926.

Mathies, A. W., Hodgman, J., and Ivler, D. (1965). Haemophilus influenzae meningitis in a premature infant. Pediatrics, 35, 791.

Mpairwe, Y. (1972). Detection of $H$. influenzae type B bactericidal antibodies. Fournal of Pediatrics, 80, 1064.

Zinner, S. H., McCormack, W. M., Lee, Y-H., Zuckerstratter, M. H., and Daly, A. K. (1972). Puerperal bacteremia and neonatal sepsis due to Haemophilus parainfluenzae: report of a case with antibody titers. Pediatrics, 49, 612.

Sheila Nicholls, T. D. Yuille, and R. G. Mitchell

Departments of Paediatrics and Pathology, John Radcliffe Hospital, Oxford.

Correspondence to Dr. R. G. Mitchell, Department of Pathology, Churchill Hospital, Oxford.

\section{Diazepam and active metabolite in breast milk and their transfer to the neonate}

Diazepam has for many years been widely used in labour and the puerperium. It is known that it can be secreted into breast milk, and if it is administered during labour and afterwards appreciable amounts may be transferred to the neonate who can only slowly metabolize it. Diazepam and its active metabolite, desmethyldiazepam, can be detected 10 days after single administration of the drug to the mother (Eliot et al., 1975). Drowsiness in neonates has been attributed to diazepam and its active metabolites in breast milk
(Patrick, Tilstone, and Reavy, 1972). A maternal concentration to milk concentration ratio of 10:1 for each compound has been reported (Erkkola and Kanto, 1972).

The relation between substances in the mother's blood and its transfer to breast milk is complex and related, not only to simple diffusion but also to ionization of the drug and the protein and fat binding properties of the substances (Rasmussen, 1973). Diazepam is highly protein bound and only the unbound fraction can pass through biological membranes. Ionized fractions will diffuse, however, and nonionized fractions will pass into milk.

\section{Materials and methods}

Nine breast feeding mothers were given diazepam for post-partum tranquillization of persistent hypertension, and maternal milk and simultaneous blood samples were obtained. Capillary blood samples were taken from the infants coincident with routine neonatal investigations and all samples were analysed by electroncapture gas-liquid chromatography (Baird, Hailey, and Malcolm, 1973). The infants were also closely observed for the clinical features associated with diazepam toxicity.

\section{Results}

Administration of diazepam during the week before delivery, the day of delivery, and during lactation were considered separately. The maximum maternal blood level of diazepam and desmethyldiazepam and the level in the breast milk, together with the level found in infant blood, were estimated and compared.

No adverse clinical affects were observed other than 3 cases of mild jaundice. This incidence was not thought to be exceptional. Two typical cases are represented in Fig. 1 and 2. The persistence of

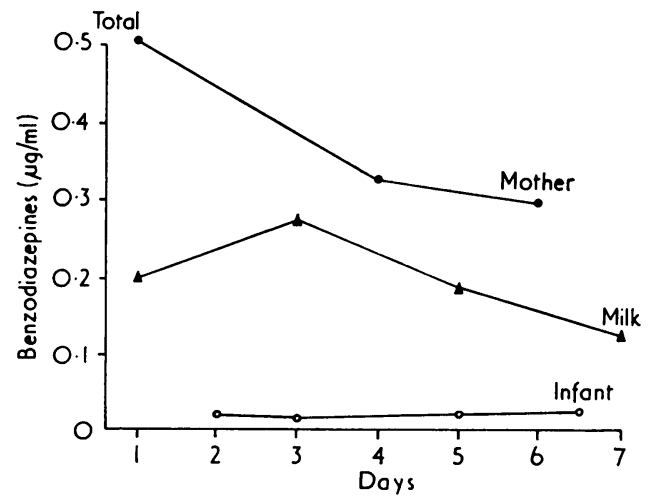

FIG. 1.-Diazepam and desmethyldiazepam levels in infant blood after a single maternal dose of diazepam. 